ITEP-TH-8/21

IITP-TH-5/21

\title{
Integrable extensions of classical elliptic integrable systems
}

\author{
M. Olshanetsky \\ NRC Kurchatov Institute, ITEP, Moscow, B. Cheremushkinskaya, 25, Moscow, 117259, Russia \\ Institute for Information Transmission Problems RAS (Kharkevich Institute), \\ Bolshoy Karetny per. 19, Moscow, 127994, Russia \\ Moscow Institute of Physics and Technology, \\ Inststitutskii per. 9, Dolgoprudny, 141700 Moscow Region, Russia \\ Email olshanet@itep.ru
}

In Memory of Mikhail Konstantinovich Polivanov

\begin{abstract}
In this article we consider two particular examples of general construction proposed in arXiv:2012.15529, We consider the integrable extensions of the classical elliptic CalogeroMoser model of $\mathrm{N}$ particles with spin and the integrable Euler-Arnold top related to the group SL $(\mathrm{N}, \mathrm{C})$. The extended systems has additional N-1 degrees of freedom and can be described in terms of the Darboux variables.
\end{abstract}

Keywords: Hitchin systems, Calogero-Moser model, Euler-Arnold top

\section{Introduction and summary}

It is possible to extend the phase space of the integrable such that an integrable system exists on the extended phase space. The extension is non-trivial if the extended system is not a direct product of two non-interacting systems. We have not a general theory of such extensions, and consider some examples based on the extensions of Hitchin type systems [1, 2]. In our construction the underlying system is the symplectic quotient of the extended system with respect to the action of some abelian group.

A particular example of a non-trivial extension that does not, however, fit into our scheme is the Hitchin systems related to parabolic Higgs bundles (the Hitchin systems with spin variables). The symplectic quotients of these systems are the Hitchin systems defined on smooth curves (the systems without spin.

An example of such a non-trivial expansion is the passage from the system of $N$ CalogeroMoser (CM) particles, to the same system of particles equipped with spin [3, 4]. Here, in particular, we consider the further extension of this system to the system with additional $N-1$ degrees of freedom.

In our previous publication [5] we proposed some generalizations of the parabolic Higgs bundles which lead to a special extensions of integrable systems. The advantage of our construction is the existence of Darboux coordinates on the extended system. 
The first example of these systems is the Sutherland model two types of spins [6, 7]. Here we consider in details two additional examples. The first one is the extension of integrable EulerArnold top related to the group $\operatorname{SL}(N, \mathbb{C})[8,9]$. The second is the extension of spin elliptic CM system.

The next chapter is auxiliary. We describe the symplectic structure on one of the symmetric $\mathrm{SL}(N, \mathbb{C})$-spaces and on the $\mathrm{SL}(N, \mathbb{C})$-coorbits and establish the relation between them. In the next chapter we actually consider the above two examples

\section{Cotangent bundles to symmetric spaces and coadjoint orbits}

\subsection{Cotangent bundles to groups.}

Consider the complex groups $\operatorname{GL}(N, \mathbb{C}), \operatorname{SL}(N, \mathbb{C})$ and $\operatorname{PGL}(N, \mathbb{C})$ for $N \geq 2$.

$$
\begin{gathered}
\operatorname{GL}(N, \mathbb{C})=\left\{f \in \operatorname{Mat}_{N} \mid \operatorname{det} f \neq 0\right\}, \\
\operatorname{SL}(N, \mathbb{C})=\{f \in \operatorname{GL}(N, \mathbb{C}) \mid \operatorname{det} f=1\}, \operatorname{SL}(N, \mathbb{C}) \subset \operatorname{GL}(N, \mathbb{C}), \\
\operatorname{PGL}(N, \mathbb{C})=\operatorname{GL}(N, \mathbb{C}) / \mathbb{C}^{*},
\end{gathered}
$$

where $\mathbb{C}^{*}$ is the center of $\operatorname{GL}(N, \mathbb{C})$. Let $\mathcal{Z}=\mathbb{Z}_{N}$ be the center of $\operatorname{SL}(N, \mathbb{C})$. Then

$$
\operatorname{PGL}(N, \mathbb{C})=\operatorname{SL}(N, \mathbb{C}) / \mathcal{Z}
$$

Let $G$ be the group $\operatorname{GL}(N, \mathbb{C}), \operatorname{SL}(N, \mathbb{C})$ or $\operatorname{PGL}(N, \mathbb{C})$ and $\mathfrak{g}$ their Lie algebras. We identify $\mathfrak{g}$ with the Lie coalgebra $\mathfrak{g}^{*}$ by means the invariant metric $($,$) on \mathfrak{g}$. Then the algebra $\mathfrak{g}$ becomes a Poisson space. Let $\mathbf{S}=\sum S^{a} T_{a}$ be the expansion in a basis of $\mathfrak{g}$. The Poisson brackets are the Lie-Poisson brackets

$$
\left\{S^{a}, S^{b}\right\}=C_{c}^{a b} S^{c},
$$

where $C_{c}^{a b}$ are structure constants of the algebra $\mathfrak{g}$. These brackets are degenerated on $\mathfrak{g}$. Fixing $l=\operatorname{rank} \mathfrak{g}$ Casimir functions

$$
(\mathbf{S})=c_{1},\left(\mathbf{S}^{2}\right)=c_{2}, \ldots,\left(\mathbf{S}^{N}\right)=c_{N}
$$

we come to the non-degenerated brackets on co-adjoint orbits $\mathcal{O}_{\nu}$ described below. The dimension of the generic orbit is $\operatorname{dim}\left(\mathcal{O}_{\nu}\right)=\operatorname{dim} G-l$. In what follows we will consider only these types of orbits.

The cotangent bundle $T^{*} G$ is identified with the tangent bundle $T G$. Using its trivialization we describe $T^{*} G$ as

$$
T^{*} G=\mathfrak{g} \times G=\{\zeta \in \mathfrak{g}, g \in G\} .
$$

The canonical invariant symplectic form on $T^{*} G$ in these coordinates takes the form

$$
\omega=D\left(\zeta, D g g^{-1}\right)=\left(\mathbf{X}, g^{-1} D g\right),
$$

where

$$
\mathbf{X}=g^{-1} \zeta g \in \mathfrak{g} .
$$

The form is invariant under the left action of subgroup $K \subseteq G$

$$
\zeta \rightarrow f \zeta f^{-1}, g \rightarrow f g, \mathbf{X} \rightarrow \mathbf{X}, f \in K
$$

producing the left moment

$$
\mu^{L}(\zeta, g)=\left.\zeta\right|_{\mathfrak{k}^{*}}=\left.g \mathbf{X}^{-1}\right|_{\mathfrak{k}^{*}},
$$


where $\mathfrak{k}=\operatorname{Lie}(K)$, and $\mathfrak{k}^{*}$ is the Lie coalgebra.

The right action

$$
\zeta \rightarrow \zeta, g \rightarrow g f^{-1}, f \in G
$$

leads to the moment map

$$
\mu^{R}(\zeta, g)=g^{-1} \zeta g=\mathbf{X}
$$

Consider the interrelations between $T^{*} \mathrm{GL}(N, \mathbb{C})$ and $T^{*} \mathrm{SL}(N, \mathbb{C})$. The symplectic form $\omega$ (2.6) on $T^{*} \mathrm{GL}(N, \mathbb{C})$ is invariant under the scaling

$$
g \rightarrow g \lambda, \zeta \rightarrow \zeta, \lambda \in \mathbb{C}^{*}
$$

The corresponding moment is $\operatorname{tr} \zeta$. We fix the gauge by the condition det $g=1$ (2.1). In this way we come to $T^{*} \mathrm{SL}(N, \mathbb{C})$ as the symplectic quotient

$$
T^{*} \mathrm{SL}(N, \mathbb{C})=\mathbb{C}^{*} \backslash \backslash T^{*} \mathrm{GL}(N, \mathbb{C})=\{(\zeta, g) \mid \operatorname{tr} \zeta=0, \operatorname{det} g=1\} .
$$

The condition det $g=1$ does not fix the gauge completely. One can further act by the center $\mathcal{Z}$ on $g \rightarrow \gamma g$ that preserves this condition. In this way we come to the cotangent bundle $T^{*} \operatorname{PGL}(N, \mathbb{C})$.

The symplectic form on $T^{*} \mathrm{SL}(N, \mathbb{C})$ is

$$
\omega=D\left(\zeta, D g g^{-1}\right)=\left(\mathbf{X}, g^{-1} D g\right), \text { where }(\zeta, g) \text { satisfy (2.12) . }
$$

Since $\mathbf{X}$ is gauge invariant and $\operatorname{tr} \mathbf{X}=0$ it is a section of the cotangent bundle $T^{*} \operatorname{SL}(N, \mathbb{C})$.

There is a residual symmetry defined by multiplication on the center element

$$
\mathbf{e}_{N}(\gamma), \gamma=\operatorname{diag}(1, \ldots, 1), \mathbf{e}_{N}(\gamma)=\exp \frac{2 \pi \imath \gamma}{N}, \mathbf{e}_{N}(\gamma) \in \mathcal{Z}
$$

After factorizing we come to the cotangent bundle $T^{*} \operatorname{PGL}(N, \mathbb{C})$.

\subsection{Co-adjoint orbits}

Consider the cotangent bundle $T^{*} \operatorname{SL}(N, \mathbb{C})$. and the left symplectic quotient $\operatorname{SL}(N, \mathbb{C}) \backslash \backslash \nu T^{*} \operatorname{SL}(N, \mathbb{C})$. with the moment $\mu^{L}(2.9)$ taking value in the Cartan subalgebra $\mathfrak{h}^{\mathbb{C}} \subset \operatorname{sl}(N, \mathbb{C})$

$$
\mu^{L}(\zeta, g)=\zeta=\nu \in\left(\mathfrak{h}^{\mathbb{C}}\right)^{*} \sim \mathfrak{h}^{\mathbb{C}} .
$$

Here $\nu$ is a fixed regular element of $\mathfrak{h}^{\mathbb{C}}$. The subgroup preserving this value is the Cartan subgroup $H^{\mathbb{C}} \subset \mathrm{SL}(N, \mathbb{C})$. Thus, the symplectic quotient is defined as

$$
\operatorname{SL}(N, \mathbb{C}) \backslash \backslash{ }_{\nu} T^{*} \operatorname{SL}(N, \mathbb{C})=\left\{(\zeta, g) \mid \zeta=\nu \in \mathfrak{h}^{\mathbb{C}}, g \sim f g, f \in H^{\mathbb{C}}\right\} .
$$

It is the co-adjoint orbit $\mathcal{O}_{\nu}$

$$
\mathcal{O}_{\nu}=H^{\mathbb{C}} \backslash \mathrm{SL}(N, \mathbb{C})=\left\{\mathbf{S}=g^{-1} \nu g \mid g \in \mathrm{SL}(N, \mathbb{C}), \nu \in \mathfrak{h}^{\mathbb{C}}\right\} .
$$

After substituting $\zeta=\nu(2.15)$ in $(2.6)$ the form $\omega$ on $T^{*} \operatorname{SL}(N, \mathbb{C})$ becomes the KirillovKostant on $\mathcal{O}_{\nu}$ form

$$
\omega^{K K}=D\left(\nu, D g g^{-1}\right) .
$$

The form is invariant under the right $G^{\mathbb{C}}$ action (2.8). This transformation generates the moment

$$
\mu^{R}=g^{-1} \nu g=\mathbf{S} .
$$


For $\mathfrak{g}=\operatorname{sl}(N, \mathbb{C})$ the Casimir functions (2.4) are

$$
\left(\mathbf{S}^{2}\right)=c_{2}, \ldots,\left(\mathbf{S}^{N}\right)=c_{N},\left(c_{2}=\sum_{j=1}^{N} \nu_{j}^{2}, \ldots, c_{N}=\sum_{j=1}^{N} \nu_{j}^{N}\right)
$$

and we assume that $c_{j} \neq c_{k}$ for $j \neq k$. The corresponding orbit is

$$
\mathcal{O}_{\nu}=g^{-1} \nu g, g \in \mathrm{SL}(N, \mathbb{C}), \nu=\operatorname{diag}\left(\nu_{1}, \ldots, \nu_{N}\right), \operatorname{tr} \nu=0,
$$

and $\nu_{j} \neq \nu_{k}$ for $j \neq k$. The dimension of the generic orbit is

$$
\operatorname{dim}_{\mathbb{C}}\left(\mathcal{O}_{\nu}\right)=\operatorname{dim}_{\mathbb{C}} \operatorname{SL}(N, \mathbb{C})-(N-1)=N(N-1) .
$$

\subsection{Cotangent bundles to $T^{*}(\mathrm{SO}(N, \mathbb{C}) \backslash \mathrm{GL}(N, \mathbb{C}))$}

Consider the group $G=\mathrm{GL}(N, \mathbb{C})$, its subgroup

$$
K=\mathrm{SO}(N, \mathbb{C})=\left\{g \in \mathrm{GL}(N, \mathbb{C}) \mid g^{T} g=I d, \operatorname{det} g=1\right\}
$$

and the quotient space $\mathcal{X}^{G}=\mathrm{SO}(N, \mathbb{C}) \backslash \mathrm{GL}(N, \mathbb{C})$. Thereby $\mathcal{X}^{G}=\{x\}$ is the space of complex symmetric matrices $x$ with det $x \neq 0$. Evidently, the element

$$
\mathcal{Q}=g^{T} g, g \in \mathrm{GL}(N, \mathbb{C})
$$

is an element of $\mathcal{X}^{G}$.

The cotangent bundle $T^{*} \mathcal{X}^{G}=T^{*}(\mathrm{SO}(N, \mathbb{C}) \backslash \mathrm{GL}(N, \mathbb{C}))$ can be identified with the symplectic quotient $\mathrm{SO}(N, \mathbb{C}) \backslash \backslash T^{*} G$. The form $\omega(2.6)$ on $T^{*} \mathrm{GL}(N, \mathbb{C})$ is invariant under the left $\mathrm{SO}(N, \mathbb{C})$-action

$$
\zeta \rightarrow f \zeta f^{-1}, g \rightarrow f g, f \in \mathrm{SO}(N, \mathbb{C}) .
$$

The corresponding moment map assumes the form $\mu^{L}=\left.\zeta\right|_{\mathrm{so}(N, \mathbb{C})}$ and we take

$$
\left.\zeta\right|_{\mathrm{so}(N, \mathbb{C})}=0 .
$$

Note that the $\mathrm{SO}(N, \mathbb{C})$-action preserves the moment constraint $\left.f \zeta f^{-1}\right|_{\mathrm{so}(N, \mathbb{C})}=0$ for $f \in$ $\mathrm{SO}(N, \mathbb{C})$.

The Lie algebra $\mathfrak{g}=\operatorname{gl}(N, \mathbb{C})$ can be decomposed in the sum of the antisymmetric and symmetric matrices

$$
\operatorname{gl}(N, \mathbb{C})=\operatorname{so}(N, \mathbb{C})+\mathfrak{p}^{G} .
$$

The space of symmetric matrices $\mathfrak{p}^{g}$ can be considered as the tangent space to $\mathcal{X}^{G}=\{\mathrm{SO}(N, \mathbb{C}) g\}$ at the point $g=I d$. These subspaces are orthogonal with respect to the invariant metric on $\operatorname{gl}(N, \mathbb{C})$. Its restriction on $\mathfrak{p}^{G}$ is non-degenerate.

The moment condition (2.26) means that $\zeta \in \mathfrak{p}^{G}$. In this way the symplectic quotient $\mathrm{SO}(N, \mathbb{C}) \backslash \backslash T^{*} \mathrm{GL}(N, \mathbb{C})$ is defined by the set of pairs $(g, \zeta)$ that satisfy the equivalence relation

$$
\left\{(g, \zeta) \sim\left(f g, f \zeta f^{-1}\right), f \in \mathrm{SO}(N, \mathbb{C}), g \in \mathrm{GL}(N, \mathbb{C}) \zeta \in \mathfrak{p}^{G}\right\}
$$

Since $g \in \operatorname{GL}(N, \mathbb{C})$ is defined up to the left multiplication by $\mathrm{SO}(N, \mathbb{C})$, we come to the cotangent bundle $T^{*} \mathcal{X}^{G}, \mathcal{X}^{G}=\mathrm{SO}(N, \mathbb{C}) \backslash \mathrm{GL}(N, \mathbb{C})$

$$
T^{*} \mathcal{X}^{G}=\mathrm{SO}(N, \mathbb{C}) \backslash \backslash T^{*} \mathrm{GL}(N, \mathbb{C})
$$

It follows from this definition of $T^{*} \mathcal{X}^{G}$ that

$$
\operatorname{dim} T^{*} \mathcal{X}^{G}=2(\operatorname{dim} \operatorname{GL}(N, \mathbb{C})-\operatorname{dim} \operatorname{SO}(N, \mathbb{C}))=N(N+1) .
$$


The symplectic form on $T^{*} \mathcal{X}^{G}$ coincides with (2.6)

$$
\omega=D\left(\zeta, D g g^{-1}\right)
$$

where the pair $(\zeta, g)$ satisfies (2.28).

Let $g \in \mathrm{GL}(N, \mathbb{C})$ and $\zeta \in \mathfrak{p}^{G}$. Consider the element

$$
\mathbf{X}^{G}=g^{-1} \zeta g \in \operatorname{gl}(N, \mathbb{C}) .
$$

It is a section of the bundle $T^{*} \mathcal{X}^{G}$. In this terms $\omega$ (2.31) assumes the form

$$
\omega=D\left(\mathbf{X}^{G}, g^{-1} D g\right) .
$$

Since $\mathbf{X}^{G} \in \operatorname{gl}(N, \mathbb{C})$ it can be expanded in the basis $T_{a}$ of the algebra $\operatorname{gl}(N, \mathbb{C})$

$\mathbf{X}^{G}=\sum X^{a} T_{a}$. The coefficients $X^{a}$ form the Lie-Poisson algebra on $\operatorname{gl}(N, \mathbb{C})$. As in (2.3)

$$
\left\{X^{a}, X^{b}\right\}=C_{c}^{a b} X^{c},\left(a, b=1, \ldots, N^{2}\right) .
$$

As above, these brackets have $N$ Casimir functions $c_{j}=\left(\mathbf{X}^{j}\right),(j=1, \ldots, N)$. But they are not the Casimir functions of the Poisson algebra on $T^{*} \mathcal{X}^{G}$.

It follows from (2.25) that $T^{*} \mathcal{X}^{G}$ can be described by the gauge-invariant variables

$$
\mathcal{P}=g^{-1} \zeta\left(g^{T}\right)^{-1}, \mathcal{Q}=g^{T} g,(2.24)
$$

where $(\zeta, g)$ satisfies (2.28) . Note that $\mathcal{P}^{T}=\mathcal{P}$ and $\mathcal{Q}^{T}=\mathcal{Q}\left(\mathcal{Q} \in \mathcal{X}^{G}\right)$. In these variables

$$
\mathbf{X}^{G}=\mathcal{P} \mathcal{Q}
$$

Let $e_{j}$ be a basis in $\mathfrak{p}^{G}\left(j=1, \ldots, \operatorname{dim} \mathfrak{p}^{G}\right)$ with the pairing $\left(e_{j}, e_{k}\right)=\delta_{j k}$. Expand $\mathcal{Q}$ and $\mathcal{P}$ in this basis $\mathcal{Q}=\sum_{j} \mathcal{Q}^{j} e_{j}, \mathcal{P}=\sum_{j} \mathcal{P}^{j} e_{j}$. In these variables the Poisson algebra on $T^{*} \mathcal{X}$ is canonical

$$
\left\{\mathcal{P}^{j}, \mathcal{Q}^{k}\right\}=\delta^{j k},(j, k=1, \ldots, \operatorname{dim} \mathfrak{k}) .
$$

To prove it define another invariant symplectic form on $T^{*} \mathcal{X}^{G}$

$$
\omega^{X^{G}}=\omega(\zeta, g)-\omega\left(\zeta,\left(g^{T}\right)^{-1}\right),
$$

where $\omega$ is (2.31). By direct calculations we find that

$$
\omega^{X^{G}}=(D \mathcal{P}, D \mathcal{Q}) .
$$

In this way we come to the Darboux brackets (2.37).

The symplectic form $\omega$ (2.33) on $T^{*} \mathcal{X}^{G}$ as well $\omega^{X}(2.39)$ is invariant under the right action of the group $G$ (2.10). Similarly to (2.9) the moment corresponding to the action is

$$
\mu^{R}(\zeta, g)=\left.g^{-1} \zeta g \in \mathfrak{g}^{\mathbb{C}}\right|_{\operatorname{Lie}^{*}(G)},
$$

$($ see $(2.26))$, or

$$
\mu^{R \stackrel{2.32}{=}} \mathbf{X}^{G \stackrel{2.36}{=}} \mathcal{P} \mathcal{Q} .
$$




\subsection{Cotangent bundle $T^{*}(\mathrm{SO}(N, \mathbb{C}) \backslash \mathrm{SL}(N, \mathbb{C}))$ and coadjoint orbits}

In what follows we need the quotient space $\mathcal{X}^{S}=\mathrm{SO}(N, \mathbb{C}) \backslash \mathrm{SL}(N, \mathbb{C})$. It is the space of complex symmetric matrices with $\operatorname{det} g=1$

$$
\mathcal{X}^{S}=\left\{g \in \mathrm{SL}(N, \mathbb{C}) \mid g^{T}=g\right\} .
$$

In fact, it is a symmetric pseudo-riemannian space (see definitions in [10, 11]).

Similarly to (2.28) the cotangent bundle $T^{*} \mathcal{X}^{S}$ is the result of the symplectic reduction of the cotangent bundle $T^{*} \mathrm{SL}(N, \mathbb{C})$ under the left action of the subgroup $\mathrm{SO}(N, \mathbb{C})$

$$
T^{*} \mathcal{X}^{S}=T^{*}(\mathrm{SO}(N, \mathbb{C}) \backslash \mathrm{SL}(N, \mathbb{C}))=\mathrm{SO}(N, \mathbb{C}) \backslash \backslash T^{*} \mathrm{SL}(N, \mathbb{C}) .
$$

Let $\mathfrak{p}^{S}$ be the subspace in the Lie algebra $\operatorname{sl}(N, \mathbb{C})$ orthogonal to the Lie subalgebra $\operatorname{so}(N, \mathbb{C})$

$$
\operatorname{sl}(N, \mathbb{C})=\operatorname{so}(N, \mathbb{C})+\mathfrak{p}^{S}, \mathfrak{p}^{S}=\left\{\zeta \in \operatorname{sl}(N, \mathbb{C}) \mid \zeta^{T}=\zeta, \operatorname{tr} \zeta=0\right\}
$$

In these terms the cotangent bundle $T^{*} \mathcal{X}^{S}$ can be identified with the set of pairs

$$
T^{*} \mathcal{X}^{S}=\left\{(g, \zeta), g \in \mathcal{X}^{S}(\underline{2.42}), \zeta \in \mathfrak{p}^{s}\right\}
$$

In this way the symplectic form $\omega$ on $T^{*} \mathcal{X}^{S}$ is

$$
\omega^{S}=D\left(\zeta, D g g^{-1}\right)=D\left(\mathbf{X}^{S}, g^{-1} D g\right), g \in \mathcal{X}^{S}, \mathbf{X}=g^{-1} \zeta g
$$

It follows from (2.30) that

$$
\operatorname{dim} T^{*} \mathcal{X}^{S}=N(N+1)-2=\operatorname{dim} \mathcal{O}_{\nu}+2(N-1)
$$

In terms of Darboux variables $\mathbf{X}^{S}=\mathcal{P} \mathcal{Q}(2.32)$, where $\mathcal{P}$ and $\mathcal{Q}$ are complex symmetric matrices with det $g=1$ and $\operatorname{tr} \mathbf{X}^{S}=0$.

Consider the symplectic action of $\mathbb{C}^{*}$ on $T^{*} \mathcal{X}^{G}$

$$
(g, \zeta) \rightarrow(\lambda g, \zeta)
$$

Since this action commutes with the $\mathrm{SO}(N, \mathbb{C})$-action we have as in (2.12) another realization of $T^{*} \mathcal{X}^{S}$

$$
T^{*} \mathcal{X}^{S}=\mathbb{C}^{*} \backslash \backslash T^{*} \mathcal{X}^{G}
$$

The Darboux variables have the form (2.35)

$$
\mathcal{P}=g^{-1} \zeta\left(g^{T}\right)^{-1}, \mathcal{Q}=g^{T} g,, \operatorname{det} g=1, \operatorname{tr} \zeta=0
$$

the form is

$$
\omega^{X^{S}}=\omega^{S}(\zeta, g)-\omega^{S}\left(\zeta,\left(g^{T}\right)^{-1}\right),
$$

where $\omega^{S}$ is (2.44) and

$$
\omega^{X^{S}}=(D \mathcal{P}, D \mathcal{Q}) .
$$

The additional constraints in these terms are

$$
\text { 1. } \operatorname{tr} \mathbf{X}^{S}=\operatorname{tr} \mathcal{P} \mathcal{Q}=0,2 . \operatorname{det} \mathcal{Q}=1 .
$$

Since the Cartan subgroup $H^{\mathbb{C}} \subset \mathrm{SL}(N, \mathbb{C})$ lies in $\mathcal{X}^{S}\left(H^{\mathbb{C}} \notin \mathrm{SO}(N, \mathbb{C})\right)$ one can consider the additional left action of $H^{\mathbb{C}}$ on $T^{*} \mathcal{X}^{S}=\operatorname{SL}(N, \mathbb{C}) \backslash \backslash \nu \mathrm{SL}(N, \mathbb{C})$

$$
g \rightarrow f g, \zeta \rightarrow f \zeta f^{-1}, f \in H^{\mathbb{C}}
$$


Let $\mathfrak{h}^{\mathbb{C}} \subset \operatorname{sl}(N, \mathbb{C})$ be the Cartan subalgebra $\mathfrak{h}^{\mathbb{C}}=\operatorname{Lie}\left(H^{\mathbb{C}}\right)$ and $\nu \in \mathfrak{h}^{\mathbb{C}}$ is a regular element. The symplectic reduction of $T^{*} \mathcal{X}^{S}$ with respect to this action is defined by the moment constraint equation

$$
\mu^{L}=\left.\operatorname{Pr} \zeta\right|_{\mathfrak{h}^{\mathbb{C}}}=\nu
$$

and the gauge invariant variable $\mathbf{X}^{S}$ (2.32). We take $\nu$ to be the same as in (2.16). Therefore $\mathbf{X}^{S}=g^{-1} \nu g \in \mathfrak{g}^{\mathbb{C}}$ is an element of the coadjoint orbit $\mathcal{O}_{\nu}$ (2.17). It means that

$$
\mathcal{O}_{\nu}=\left\{\mathbf{X}=g^{-1} \nu g \mid g \in G^{\mathbb{C}}\right\}=H^{\mathbb{C}} \backslash \backslash_{\nu} T^{*} \mathcal{X}^{S} .
$$

Summarizing we obtain the descriptions of symplectic manifolds incorporated in the commutative diagram, where the arrows mean the symplectic reductions

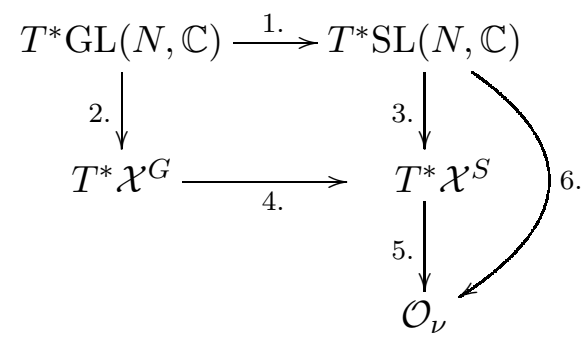

\begin{tabular}{|l|c|c|c|}
\hline \hline & Simplectic action & Constraints & Definition \\
\hline \hline 1. & $\mathbb{C}^{*} \backslash \backslash$ & $\operatorname{det} g=1, \operatorname{tr} \zeta=0$ & $(2.12)$ \\
2. & $\mathrm{SO}(N, \mathbb{C}) \backslash \backslash$ & $g^{T}=g, \zeta^{T}=\zeta$ & $(2.29)$ \\
3. & $\mathrm{SO}(N, \mathbb{C}) \backslash \backslash$ & $g^{T}=g, \zeta^{T}=\zeta$ & $(2.43)$ \\
4. & $\mathbb{C}^{*} \backslash \backslash$ & $\operatorname{det} g=1, \operatorname{tr} \zeta=0$ & $(2.46)$ \\
5. & $H^{\mathbb{C}} \backslash \backslash \nu$ & $g \in \operatorname{SL}(N, \mathbb{C}), \zeta=\nu \in \mathfrak{h}^{\mathbb{C}}$ & $(2.52)$ \\
6. & $\mathrm{SL}(N, \mathbb{C}) \backslash \backslash \nu$ & $g \in \mathcal{X}^{S}, \zeta=\nu \in \mathfrak{h}^{\mathbb{C}}$ & $(2.17)$ \\
\hline
\end{tabular}

Table

\section{Extensions of elliptic integrable systems}

Let $\Sigma_{\tau}=\mathbb{C} /(\mathbb{Z}+\tau \mathbb{Z})$ be the elliptic curve. The Lax operator $L(z)$ is a meromorphic $(1,0)$-form on $\Sigma_{\tau}$, taking values in the Lie algebra $\operatorname{sl}(N, \mathbb{C})$. It satisfies some quasi-periodicity conditions with respect to the shifts on the lattice vectors and has a simple pole at $z=0$. The residue and the quasi-periodicities fix $L(z)$. Let

$$
\left.\operatorname{Res} L(z)\right|_{z=0}=\mathbf{W} \text {. }
$$

We consider two types of the residues

$$
\mathbf{W}= \begin{cases}1 . & \mathbf{S} \in \mathcal{O}_{\nu} \\ 2 . & \mathbf{X} \in T^{*} \mathcal{X}^{G} \text { or } T^{*} \mathcal{X}^{S}\end{cases}
$$

The first case corresponds to standard integrable systems. The formula (2.45) suggests that the Lax operator in the latter case defines the extension of the standard integrable systems. We will prove that this extension is also integrable.

We consider two types of the quasi-periodicities. The first one defines the integrable EulerArnold $\operatorname{SL}(N, \mathbb{C})$ top and the second the elliptic CM system with spin. 


\subsection{Extension of integrable Euler-Arnold $\mathrm{SL}(N, \mathbb{C})$ top}

\section{Euler-Arnold $\operatorname{SL}(N, \mathbb{C})$ top}

To define the Euler-Arnold top introduce a special basis in the groups $\operatorname{GL}(N, \mathbb{C}), \operatorname{SL}(N, \mathbb{C})$ and the corresponding Lie algebras. Consider two matrices

$$
\begin{gathered}
Q=\operatorname{diag}\left(\mathbf{e}_{N}(1), \ldots, \mathbf{e}_{N}(m), \ldots, 1\right), \mathbf{e}_{N}(z)=\exp \left(\frac{2 \pi i}{N} z\right) \\
\Lambda=\left(\begin{array}{ccccc}
0 & 1 & 0 & \cdots & 0 \\
0 & 0 & 1 & \cdots & 0 \\
\vdots & \vdots & \ddots & \ddots & \vdots \\
0 & 0 & 0 & \cdots & 1 \\
1 & 0 & 0 & \cdots & 0
\end{array}\right)
\end{gathered}
$$

Note that $Q^{N}=\Lambda^{N}=I d$ and

$$
Q^{m} \Lambda^{n}=\mathbf{e}_{N}(-m n) \Lambda^{n} Q^{m}
$$

Consider two-dimensional lattices in $\mathbb{C}$

$$
\Gamma_{N}=\mathbb{Z}_{N}^{(2)}=(\mathbb{Z} / N \mathbb{Z} \oplus \mathbb{Z} / N \mathbb{Z}), \tilde{\Gamma}_{N}=\tilde{\mathbb{Z}}_{N}^{(2)}=\mathbb{Z}_{N}^{(2)} \backslash(0,0)
$$

The matrices $Q^{a_{1}} \Lambda^{a_{2}}, a=\left(a_{1}, a_{2}\right) \in \mathbb{Z}_{N}^{(2)}$ generate a basis in the group $\operatorname{GL}(N, \mathbb{C})$ and the algebra $\operatorname{gl}(N, \mathbb{C})$, while $Q^{\alpha_{1}} \Lambda^{\alpha_{2}}, \alpha=\left(\alpha_{1}, \alpha_{2}\right) \in \tilde{\mathbb{Z}}_{N}^{(2)}$ generate a basis in the Lie algebra $\operatorname{sl}(N, \mathbb{C})$. More exactly, define the generators

$$
T_{a}=\frac{N}{2 \pi \imath} \mathbf{e}_{N}\left(\frac{a_{1} a_{2}}{2}\right) Q^{a_{1}} \Lambda^{a_{2}} .
$$

They are almost invariant on the lattice $\mathbb{Z} \oplus \mathbb{Z}: T_{a_{1}+N, a_{2}+N}= \pm T_{a_{1}, a_{2}}$. Therefore, $T_{-a_{1},-a_{2}}=$ $\pm T_{N-a_{1}, N-a_{2}}$.

From (3.5) one finds the multiplication in $\operatorname{GL}(N, \mathbb{C})$

$$
T_{a} T_{b}=\kappa_{a, b} T_{a+b}, \quad \kappa_{a, b}=\frac{N}{2 \pi i} \mathbf{e}_{N}\left(-\frac{a \times b}{2}\right),\left(a \times b=a_{1} b_{2}-a_{2} b_{1}\right) .
$$

Similarly, the commutation relations for the algebra $\operatorname{sl}(N, \mathbb{C})$ in the basis $T_{\alpha}, \alpha \in \tilde{\Gamma}_{N}$ assume the form

$$
\left[T_{\alpha}, T_{\beta}\right]=\mathbf{C}(\alpha, \beta) T_{\alpha+\beta}, \mathbf{C}(\alpha, \beta)=\frac{N}{\pi} \sin \frac{\pi}{N}(\alpha \times \beta)
$$

Define invariant form on $\operatorname{gl}(N, \mathbb{C})$

$$
\left(T_{a}, T_{b}\right)=\frac{4 \pi^{2}}{N^{2}} \operatorname{tr}\left(T_{a} \cdot T_{b}\right)=\delta_{a+b, 0} .
$$

Consider the expansion of the elements of an element $\mathbf{S} \in \operatorname{sl}(N, \mathbb{C})$

$$
\mathbf{S}=\sum_{\alpha \in \tilde{\Gamma}_{N}} S_{\alpha} T_{\alpha}
$$

From (3.9) the corresponding Poisson brackets are

$$
\left\{S_{\alpha}, S_{\beta}\right\}=\mathbf{C}(\alpha, \beta) S_{\alpha+\beta} .
$$

Fix the Casimir functions (2.20). It means that $\mathbf{S}$ belongs to the corresponding coadjoint $\mathcal{O}_{\nu}$ (2.17). 
Let $\wp(x)$ be the Weierstrass function (3.17) and $\wp_{\alpha}=\wp\left(\frac{\alpha_{1}+\alpha_{2} \tau}{N}\right),\left(\alpha \in \tilde{\Gamma}_{N}\right)$. The integrable Euler-Arnold $\mathrm{SL}(N, \mathbb{C})$-top is defined by the Hamiltonian

$$
H=\frac{1}{2} \sum_{\alpha \in \tilde{\Gamma}_{N}} S_{\alpha \wp_{\alpha}} S_{-\alpha}
$$

and the Lie-Poisson brackets (3.11). The phase space $\mathcal{M}^{E T}$ of the elliptic top is the coadjoint orbit

$$
\mathcal{M}^{E T} \sim \mathcal{O}_{\nu}
$$

To find the commuting integrals of motion we use the Lax operator.

\section{Lax operator}

In this case the Lax operator $L(z)$ has the quasi-periodicities

$$
L^{E T}(z+1)=Q L^{E T}(z) Q^{-1}, L^{E T}(z+\tau)=\Lambda L^{E T}(z) \Lambda^{-1} .
$$

It can be defined in terms of the Kronecker function. The Kronecker function $\phi(u, z)$ is related to the elliptic curve $\Sigma_{\tau}$ and takes the form

$$
\phi(u, z)=\frac{\vartheta(u+z) \vartheta^{\prime}(0)}{\vartheta(u) \vartheta(z)},
$$

where $\vartheta(z)$ is the theta-function

$$
\vartheta(z \mid \tau)=q^{\frac{1}{8}} \sum_{n \in \mathbf{Z}}(-1)^{n} e^{\pi i(n(n+1) \tau+2 n z)} .
$$

In addition we need the Eisenstein function

$$
E_{1}(z)=\partial_{z} \log \vartheta(z) .
$$

The $\eta_{1}$ constant is extracted from the asymptotic of $E_{1}(z)$

$$
E_{1}(z) \sim \frac{1}{z}+\eta_{1} z+\ldots .
$$

The Weierstrass function $\wp$ is related to $E_{1}(z)$ as

$$
\wp(u)=-\partial_{z} E_{1}(u)-2 \eta_{1}
$$

is double-periodic function with a second order pole at $z=0$.

The basis of the doubly periodic functions on $\Sigma_{\tau}$ are

$$
\left\{1,\left(-\partial_{z}\right)^{k-2} \wp(z), k=2,3, \ldots\right\} .
$$

They have poles of orders $0,2,3, \ldots$.

The Kronecker function has the following quasi-periodicities:

$$
\phi(u, z+1)=\phi(u, z), \phi(u, z+\tau)=e^{-2 \pi \imath u} \phi(u, z) .
$$

and has the first order pole at $z=0$

$$
\phi(u, z) \sim \frac{1}{z}+E_{1}(u)+\frac{1}{2} z\left(E_{1}^{2}(u)-\wp(u)\right)+\ldots .
$$

It is related to the Weierstrass function $\wp$ as follows:

$$
\phi(u, z) \phi(-u, z)=\wp(z)-\wp(u) .
$$


Let

$$
\varphi_{m}(z)=\mathbf{e}_{N}\left(-m_{2} z\right) \phi\left(-\frac{m_{1}+m_{2} \tau}{N}, z\right),\left(m=\left(m_{1}, m_{2}\right)\right) .
$$

Then

$$
\varphi_{m}(z+1)=\mathbf{e}_{N}\left(-m_{2}\right) \varphi_{m}(z), \varphi_{m}(z+\tau)=\mathbf{e}_{N}\left(m_{1}\right) \varphi_{m}(z) .
$$

The Lax operator assumes the form

$$
L^{E T}(z)=\sum_{\alpha \in \tilde{\Gamma}_{N}} S_{\alpha} \varphi_{\alpha}(z) T_{\alpha}
$$

It follows from (3.19), (3.8) that $L(z)$ has needed quasi-periodicities (3.14). Note, that there are no constant terms because it does not satisfy (3.14). From (3.20)

$$
\left.\operatorname{Res} L^{E T}(z)\right|_{z=0}=\sum_{\alpha \in \tilde{\Gamma}_{N}} S_{\alpha} T_{\alpha} .
$$

These properties imply that $\left(L(z)^{k}\right)$ are doubly periodic functions with the poles up to the order k. Thereby, they can be expanded in the basis of the Weierstrass function and its derivatives (3.18)

$$
\left(\left(L^{E T}\right)^{k}(z)\right)=I_{0, k}+I_{2, k} \wp(z)+\ldots+I_{k, k}\left(\partial_{z}\right)^{k-2} \wp(z) .
$$

The term $I_{1, k}$ is absent because due to the residue theorem there are no periodic functions with one simple pole on $\Sigma_{\tau}$. In particular, from (3.21)

$$
\left(\left(L^{E T}\right)^{2}(z)\right)=I_{0,2}+I_{2,2 \wp}(z), I_{0,2}=-2 H^{E T},(3.12) .
$$

Thus, there are $\frac{1}{2} N(N+1)-1$ independent quantities $I_{s, k}(k=2,3, \ldots, N, s=0,2, \ldots, k)$

$$
\begin{array}{ccccc}
I_{0, n} & I_{2, N} & \ldots & \ldots & I_{N, N} \\
\cdots & \ldots & \ldots & \ldots & \ldots \\
& I_{0,3} & I_{2,3} & I_{3,3} & \\
\multicolumn{4}{c}{} \\
\multicolumn{4}{c}{I_{0,2}} & I_{2,2}
\end{array}
$$

It follows from the existence of the classical $r$-matrix [9] they are pairwise poisson commute. Therefore, they play the role of conservation laws of elliptic top hierarchy on $\operatorname{SL}(N, \mathbb{C})$. We have a tower of $\frac{1}{2} N(N+1)-1$ independent integrals of motion. Note that $I_{k, k}=\left(\mathbf{S}^{k}\right)(k=2,3 \ldots, N)$. are the Casimir functions (2.20). By fixing their values we come to the coadjoint orbit (2.17). In this way eventually we have $\frac{1}{2} N(N-1)$ integrals of motion. The number of integrals is equal to $\frac{1}{2} \operatorname{dim} \mathcal{O}_{\nu}(2.22)$. Therefore, the elliptic top is completely integrable system.

\section{Extension of the top}

Consider the case 2. in (3.2). Replace in expansion of the Lax operator (3.23) the coefficients $S_{\alpha}$ on $X_{\alpha}$

$$
L^{E E T}(z)=\sum_{\alpha \in \tilde{\Gamma}_{N}} X_{\alpha} \varphi_{\alpha}(z) T_{\alpha},
$$

where

$$
\mathbf{X}=\sum_{\alpha \in \tilde{\Gamma}_{N}} X_{\alpha} T_{\alpha}
$$

We take here $\operatorname{tr} \mathbf{X}=X_{0}=0$ (see 1.(2.49) ). This condition corresponds to the $T^{*} \operatorname{SL}(N, \mathbb{C})$ bundle. To define the $T^{*} \mathrm{SL}(N, \mathbb{C})$-bundle one should in addition impose the constraint 2.(2.49), corresponding to the gauge fixing. In these circumstances it is convenient to work with the not a completely gauged system. In other words we will use in our constructions $\mathbf{X}^{S}$ (3.29). 
Using the expansion (3.25) we construct the tower of commuting integrals (3.27). In terms of variables $X_{c}$ we have as above $\frac{1}{2} N(N-1)$ integrals of motion. But this number is less then $\frac{1}{2} \operatorname{dim} T^{*} \mathcal{X}^{G}(2.30)$. Remember that $\mathbf{X}^{G}$ is a special section of the bundle $T^{*} \mathcal{X}^{G}$. The coordinates on $T^{*} \mathcal{X}^{G}$ are $\mathcal{P}$ and $\mathcal{Q}(2.35)$. In these terms the quantities $I_{k, k}=\left(\left(\mathbf{X}^{G}\right)^{k}\right)$ are no longer Casimir functions with respect to the Darboux brackets (2.37). With respect to these brackets we have $\frac{1}{2} N(N+1)-1$ integrals. This number is less then $\frac{1}{2} \operatorname{dim} T^{*} \mathcal{X}^{G}(2.30)$, but coincides with $\frac{1}{2} \operatorname{dim} T^{*} \mathcal{X}^{S}$ (2.45).

In terms of the Darboux coordinates the Hamiltonian is

$$
H^{E E T}=\frac{1}{2} \sum_{\alpha \in \tilde{\Gamma}_{N}} X_{\alpha \wp_{\alpha}} X_{-\alpha}=\frac{1}{2} \sum_{\alpha \in \tilde{\Gamma}_{N}}(\mathcal{P} \mathcal{Q})_{\alpha} \wp_{\alpha}(\mathcal{P} \mathcal{Q})_{-\alpha}
$$

The Hamiltonian $H^{E E T}$ is related to the Lax operator as in (3.26)

$$
\left(\left(L^{E E T}\right)^{2}\right)(z)=I_{0,2}+I_{2,2 \wp} \wp(z), I_{0,2}=-2 H^{E E T} .
$$

Let us write the equations of motion. To define $(\mathcal{P Q})_{\alpha}(2.36)$ explicitly we introduce the basis $Y_{a}$ in the the space of symmetric matrices $\mathfrak{p}^{G}$ (2.27)

$$
Y_{a}=\frac{1}{2}\left(T_{a}+T_{a}^{T}\right)=\frac{1}{2}\left(T_{a_{1}, a_{2}}+\mathbf{e}_{N}\left(a_{1} a_{2}\right) T_{a_{1},-a_{2}}\right)=\frac{N}{4 \pi \imath} \mathbf{e}_{N}\left(\frac{a_{1} a_{2}}{2}\right) Q^{a_{1}}\left(\Lambda^{a_{2}}+\Lambda^{-a_{2}}\right), a \in \Gamma_{N} .
$$

Then

$$
Y_{a} Y_{b}=\sum_{\gamma \in \tilde{\Gamma}_{N}} f_{a, b}^{\gamma} T_{\gamma}
$$

where

$$
f_{a, b}^{\gamma}=f_{a, b},(\gamma=a+b)
$$

and

$$
\begin{aligned}
f_{a, b}= & \frac{N}{2 \pi \imath} \times\left(\mathbf{e}_{N}\left(a_{1} b_{2}-a_{1} b_{2}\right)+\mathbf{e}_{N}\left(-a_{1} a_{2}-b_{1} b_{2}+a_{2} b_{1}-a_{1} b_{2}\right)+\right. \\
& \left.\mathbf{e}_{N}\left(-a_{1} a_{2}-a_{2} b_{1}-a_{1} b_{2}\right)+\mathbf{e}_{N}\left(b_{1} b_{2}+a_{1} b_{2}+a_{2} b_{1}\right)\right)
\end{aligned}
$$

There is the expabsion

$$
\begin{gathered}
\mathcal{P}=\sum_{a \in \Gamma_{N}} p_{a} Y_{a}, \mathcal{Q}=\sum_{b \in \Gamma_{N}} q_{b} Y_{b} . \\
a_{1}=0, \ldots, N-1, a_{2}=0, \ldots, N-1 .
\end{gathered}
$$

Since $\mathbf{X}=\sum_{\gamma \in \tilde{\Gamma}_{N}} X_{\gamma} T_{\gamma}, X_{\gamma}=(\mathcal{P} \mathcal{Q})_{\gamma}$

$$
X_{\gamma}=\sum_{\left(m, n \in \Gamma_{N}\right.} \mathcal{P}_{m} \mathcal{Q}_{n} f_{m, n}
$$

Because $\operatorname{tr} \mathbf{X}=0$ there are the quadratic constraints

$$
\sum_{\left(m,-m \in \Gamma_{N}\right.} \mathcal{P}_{m} \mathcal{Q}_{-m} f_{m,-m}=0 .
$$

The corresponding equations of motion are

$$
\begin{aligned}
\partial_{t} \mathcal{Q}_{a} & =\sum_{b} \mathcal{Q}_{b} f_{a, b} \wp_{a+b} f_{-a,-b} \mathcal{P}_{-m} \mathcal{Q}_{-n}, \\
\partial_{t} \mathcal{P}_{a} & =-\sum_{b} \mathcal{P}_{b} f_{a, b} \wp_{a+b} f_{-a,-b} \mathcal{P}_{-b} \mathcal{Q}_{-a} .
\end{aligned}
$$




\subsection{Extension of Calogero-Mozer system}

\section{Elliptic Calopgero-Moser system with spin}

Consider the Chevalley basis $\left\{e_{j}=E_{j j}, E_{j K}\right\}$ in the Lie algebra $\operatorname{sl}(N, \mathbb{C})$. The matrices $\mathbf{S}=\sum S_{j k} E_{j k}$ define the spin variables and the diagonal matrices $\mathbf{u}=\sum_{j} u_{j} e_{j}$ and $\mathbf{v}=\sum_{j} v_{j} e_{j}$ $\left(\sum u_{j}=\sum v_{j}=0\right)$ define the coordinates and momenta of $\mathrm{N}$ particles. They have the canonical brackets $\left\{v_{j}, u_{k}\right\}=\delta_{j k}$. The coefficients $\left(S_{j j}, S_{j k}\right)$ satisfy the Poisson-Lie algebra $\operatorname{sl}(N, \mathbb{C})$. The polynomial $c_{2}=\operatorname{tr} \mathbf{S}^{2}, \ldots, c_{N}=\operatorname{tr} \mathbf{S}^{N}$ (compare with (2.201) ) are the Casimir functions with respect to these brackets. Fixing them one can invert the brackets and come to the nondegenerate form $\omega^{K K}=\left(\mathbf{S}, g^{-1} D g\right)$ on the orbit $\mathbf{S}=g^{-1} \nu g$ (2.18). This set forms coordinates on the phase space $\tilde{\mathcal{M}}^{C M}$

$$
\tilde{\mathcal{M}}^{C M}=\{\mathbf{v}, \mathbf{u}, \mathbf{S}\} .
$$

There is residual gauge symmetry acting only on the spin variables $H^{\mathbb{C}} \in \mathrm{SL}(N, \mathbb{C})$ :

$$
g \rightarrow g h^{-1}, \mathbf{S} \rightarrow h \mathbf{S} h^{-1} .
$$

The corresponding moment constraints are $S_{j j}=0$. To come to non-degenerate brackets one should in addition fix the gauge, for example as $S_{j, j+1}=1$. The brackets for the variables $S_{j k}$ become the Dirac brackets corresponding to these constraints. Thus we come to the phase space for the elliptic CM system with spin

$$
\mathcal{M}^{C M}=\tilde{\mathcal{M}}^{C M} / / H^{\mathbb{C}}=(\mathbf{v}, \mathbf{u}) \mid,\left\{S_{j k}, j \neq k, S_{j, j+1}=1\right\} .
$$

It has dimension

$$
\operatorname{dim}_{\mathbb{C}} \mathcal{M}^{C M}=N(N-1) .
$$

Note that it coincides with $\operatorname{dim} \mathcal{O}_{\nu}(2.22)$.

The Hamiltonian of the elliptic Calopgero-Moser system with spin has the form

$$
H^{C M}=\frac{1}{2}(\mathbf{v}, \mathbf{v})+\sum_{j \neq k} S_{j k} S_{k j} \wp\left(u_{j}-u_{k}\right) .
$$

The Lax operator corresponding the Calogero-Mozer has the quasi-periodicities

$$
L^{C M}(z+1)=L^{C M}(z), L^{C M}(z+\tau)=\mathcal{R} L^{C M}(z) \mathcal{R}^{-1},
$$

where $\mathcal{R}=\operatorname{diag}\left(\mathbf{e}\left(u_{1}\right), \ldots \mathbf{e}\left(u_{N}\right)\right)$. It follows from (3.19) and (3.20) that

$$
L^{C M}(z)=\sum_{j=1}^{N} v_{j} e_{j}+\sum_{j \neq k} S_{j k} \phi\left(u_{j k}, z\right) E_{j k}, \quad u_{j k}=u_{j}-u_{k} .
$$

In terms of the Lax operator the Hamiltonian of the system (3.39) is defined as in (3.26). In the similar way we obtain $\frac{1}{2} N(N-1)-1$ integrals of motion.

\section{Extension of the system}

Assume now that the residue of the Lax operator 2.(3.2) is a section $\mathbf{X}$ (2.7) of the cotangent bundle $T^{*} \mathcal{X}^{S}$

$$
\mathbf{X}=\sum_{j=1}^{N} X_{j j} e_{j}+\sum_{j \neq k} X_{j k} E_{j k}
$$

Then as in (3.41) the corresponding Lax operator $L^{E C M}$ of the extended Calogeroo-Moser model has the form

$$
L^{E C M}(z)=\sum_{j=1}^{N} v_{j} e_{j}+\sum_{j \neq k} X_{j k} \phi\left(u_{j k}, z\right) E_{j k}, \quad, u_{j k}=u_{j}-u_{k}
$$


Here we assume that $X_{j j}=0$. These constraints come as above after the symplectic reduction by the diagonal matrices $H^{\mathbb{C}}$. The symplectic quotient is the phase space of the extended CM system (compare with (3.35)

$$
\tilde{\mathcal{M}}^{E C M}=\{\mathbf{v}, \mathbf{u}, \mathbf{X}\} .
$$

Then

$$
\begin{gathered}
\mathcal{M}^{E C M}=\tilde{\mathcal{M}}^{E C M} / / H^{\mathbb{C}}=\left\{(\mathbf{v}, \mathbf{u}), X_{j k}(j \neq k), X_{j, j+1}=1\right\} . \\
\operatorname{dim} \mathcal{M}^{E C M}=N(N+1)-2
\end{gathered}
$$

It coincides with $\operatorname{dim}_{\mathbb{C}} T^{*} \mathcal{X}^{S}(2.45)$.

The Hamiltonian of the system takes the form (see(3.39)

$$
H^{E C M}=\frac{1}{2}(\mathbf{v}, \mathbf{v})+\sum_{j \neq k} X_{j k} X_{k j} \wp\left(u_{j}-u_{k}\right) .
$$

As in the case of the elliptic top we use the expansion (3.25) to define the tower of commuting integrals (3.27). We have as above $\frac{1}{2} N(N+1)-1$ integrals of motion. The quantities $I_{k, k}=$ $\left((\mathbf{X})^{k}\right)$ are no longer Casimir functions with respect to the Darboux brackets (2.37). With respect to these brackets we have $\frac{1}{2} N(N+1)-1$ integrals. This number is less then $\frac{1}{2} \operatorname{dim} T^{*} \mathcal{X}^{G}(2.30)$, but coincides with $\frac{1}{2} \operatorname{dim} T^{*} \mathcal{X}^{S}(2.45)$.

Note that $H^{\mathbb{C}} \subset \mathrm{SL}(N, \mathbb{C})$ lies in $\mathcal{X}^{S}\left(H^{\mathbb{C}} \notin \mathrm{SO}(N, \mathbb{C})\right)$. One can consider the additional left action of the subgroup $H^{\mathbb{C}}$ on the moduli space $\mathcal{M}^{E C M}$. The subgroup does not act on the variables $\mathbf{v}$ and $\mathbf{u}$ and acts on $g \rightarrow h g$ as in (2.50). We have found that $\mathcal{O}_{\nu}=H \backslash \backslash_{\nu} T^{*} \mathcal{X}^{S}$ (2.52). Since the left action does not touch $(\mathbf{v}, \mathbf{u})$ we obtain that

$$
H^{\mathbb{C}} \backslash \backslash{ }_{\nu} \mathcal{M}^{E C M}=\mathcal{M}^{E T} \sim \mathcal{O}_{\nu},(3.13) .
$$

Describe the phase space of the extended CM system in terms of the Darboux variables. Consider the basis

$$
Y_{j k}=\frac{1}{2}\left(E_{j k}+E_{k j}\right), e_{j}=E_{j j}
$$

in the space of symmetric matrices. As in (3.33)

$$
\mathcal{P}=\sum_{j} p_{j} e_{j}+\sum_{j>k} p_{j k} Y_{j k}, \quad \mathcal{Q}=\sum_{j} q_{j} e_{j}+\sum_{j>k} q_{j k} Y_{j k} .
$$

Then

$$
\mathbf{X}=\mathcal{P} \mathcal{Q}=\sum_{a} X_{a} e_{a}+\sum_{a \neq b} X_{a b} E_{a b}
$$

Since

$$
\begin{gathered}
e_{l} Y_{j k}=f_{l j k}^{a b} E_{a b}, f_{l j k}^{a b}=\frac{1}{2}\left(\delta_{l j} \delta_{l a} \delta_{k b}+\delta_{l k} \delta_{k a} \delta_{j b}\right), \\
Y_{j k} e_{l}=f_{j k l}^{a b} E_{a b}, f_{j k l}^{a b}=\frac{1}{2}\left(\delta_{l k} \delta_{j a} \delta_{k b}+\delta_{l j} \delta_{k a} \delta_{j b}\right) \\
Y_{j k} Y_{m n}=f_{j k m n}^{a b} E_{a b}, \\
f_{j k m n}^{a b}=\frac{1}{4}\left(\delta_{a j} \delta_{b n} \delta_{k m}+\delta_{a j} \delta_{b m} \delta_{k n}+\delta_{a k} \delta_{b n} \delta_{j m}+\delta_{a k} \delta_{b m} \delta_{j n}\right) \\
f_{j k m n}^{a a}=\frac{1}{4}\left(\delta_{a j} \delta_{j n} \delta_{k m}+\delta_{a k} \delta_{k m} \delta_{k n}+\delta_{a k} \delta_{k n} \delta_{j m}+\delta_{k a} \delta_{k m} \delta_{j n}\right)
\end{gathered}
$$

we obtain

$$
X_{a}=p_{a} q_{a}+\sum_{j k m n} f_{j k m n}^{a a} p_{j k} q_{m n}
$$




$$
X_{a b}=\sum_{l j k} p_{l} q_{m n} f_{l j k}^{a b}+\sum_{j k m n} f_{j k m n}^{a b} p_{j k} q_{m n}
$$

The Hamiltonian $H^{E C M}(3.45)$ is the quartic polynomial in terms of the Darboux variables. In addition, there are the quadratic constraints $X_{a}=0$ (3.47).

Acknowledgments. The work was supported by RFBR grant 19-51-18006-BOLG

\section{References}

[1] N.Hitchin, Stable bundles and integrable systems, Duke Math. J.,54:1, (1987), 91-114.

[2] N.Nekrasov, Holomorphic bundles and many-body systems, Commun. Math. Phys., $\underline{180}$ 587-603; hep-th/9503157, (1996).

[3] J.Gibbons, and T.Hermsen, A generalization of the Calogero-Moser systems, Physica 11D , 337-348 (1984).

[4] S.Wojciechowski, An integrable marriage of the Euler equations with the Calogero-Moser systems, Phys. Lett. A, 111, 101-103 (1985).

[5] A.Levin, M.Olshanetsky, A.Zotov, Generalizations of parabolic Higgs bundles, real structures and integrability, arXiv preprint arXiv:2012.15529 (2020).

[6] L.Fehér, B.G.Pusztai, A class of Calogero type reductions of free motion on a simple Lie group, Letters in Mathematical Physics, 79(3), 263-277 (2007).

[7] S.Kharchev, A.Levin, M.Olshanetsky, A.Zotov,, Calogero-Sutherland system with two types interacting spins, JETP Letters, 느, No. 3 179-183; arXiv:1706.08793, (2017).

[8] A.Reyman, M.Semenov-Tian-Shansky, Group-theoretical methods in the theory of finitedimensional integrable systems. Dynamical systems VII, 116-225, (1994).

[9] B.Khesin, A.Levin, M.Olshanetsky, Bihamiltonian structures and quadratic algebras in hydrodynamics and on non-commutative torus, Communications in mathematical physics, 250(3), 581-612 (2004).

[10] M.Berger, Les espaces symétriques noncompacts, In Annales sci de L'ÉNS 74, (1957), No.2, 85-177.

[11] S.Helgason, Differential geometry, Lie groups and symmetric spaces, Academic press (1978). 